\title{
ESTIMATION OF SOME GENETIC PARAMETERS FOR YIELD AND ITS COMPONENTS IN SOME HULLESS BARLEY GENOTYPES UNDER NORMAL AND WATER STRESS CONDITIONS
}

\author{
Sultan, M. S. ${ }^{1}$; \\ Sally E. El-Wakeel ${ }^{2}$ \\ M. A. Abdel-Moneam ${ }^{1}$; A. A. Eid ${ }^{2}$ and
}

${ }^{1}$ Agronomy Dept., Fac. Agric., Mansoura Univ. Egypt

${ }^{2}$ Barley Dept., Field Crops Res. Institute, ARC, Egypt

\begin{abstract}
This investigation was carried out at Sakha Agricultural Research Station, ARC, Egypt during the two growing seasons (2010/2011 and 2011/2012). Seven lines and three testers were used to develop barley hybrids for yield and its components under normal and water stress conditions. Through this study, general and specific combining ability, genetic parameters, heritability and genetic advance from selection were studied for plant height $(\mathrm{cm})$, spike length $(\mathrm{cm})$, no. of spikes/plant (spike), no. of grains/spike (grain), 100-grain weight (g), grain yield/plant (g) and drought susceptibility index. Data revealed that most of the variance due to the lines, testers and line $x$ testers were highly significant for most studied traits. The estimates of GCA effect indicated that, parent $L_{6}$ considered as good combiners for no. of grains/spike, 100 -grain weight and grain yield/plant under the two conditions and their combined, spike length and no. of spikes/plant under stress condition. Also, the parent $L_{2}$ for plant height and parent $\mathrm{T}_{2}$ for 100-grain weight are considered as good combiner under the two conditions and their combined. For SCA estimates, results indicated that the crosses; 14 for plant height under normal condition, 10, 11 for spike length, 3 , 17 for no. of spikes/plant under stress condition and cross 17 for grain yield /plant under the two conditions and their combined considered as the best crosses for these traits. Heritability values in broad sense values were found to be moderate to high in magnitude for most of the studied characters. On the other side, heritability values in narrow sense were found to be low to moderate in these traits. Finally, the top crosses no. 12 and 17 conceder as the best crosses for under study, so using it in breeding program for high yielding under normal water stress would be useful.
\end{abstract}

\section{INTRODUCTION}

Barley (Hordeum Vulgare L.) has a great adaptation potential in many regions of the world. It has a good tolerance to biotic stresses such as salinity, drought, frost and heat. It is considered one of the most important crops ranking the fourth in the world cereal crops production. Its economic importance is due to its usage it for animal feeding, brewing malts and human food in some areas. In Egypt, barley is mainly used as animal feed (grain and straw) and sometimes for bread making by bedouins.

The ability of a cultivar to produce high and satisfactory yield over a wide range of stress and non-stress environments is very important. Finlay (1968) believed that stability over environments and yield potential are more or less independent of each other. Blum (1979) suggested that one method of breeding for increased performance under water stressed conditions might be 
to breed for superior yield under optimum conditions on the assumption that the best lines would also perform well under sub optimum conditions. Sojka et al. (1981) pointed out that a high yield base line that allows a cultivar to do well over a range of environments does not imply drought resistance. They defined drought tolerance as the ability to minimize yield loss in the absence of soil water availability. The ideal situation would be to have a highly stable genotype with high yield potential (Finlay \& Wilkinson, 1963; Smith, 1982).

Therefore, the main objectives of this study included the induction of new promising barley genotypes having high yield potentially and more tolerant to water stress, this was approached through the following:-

1- Identification of superior parents and their crosses from a 7 line $\times 3$ tester of barley parental genotypes under normal and water stress conditions.

2- Estimation of combining ability effects for grain yield and some related agronomic traits under normal and water stress conditions.

3- Estimation the heritability and genetic advance from selection for yield and some related agronomic traits under normal and water stress conditions.

\section{MATERIALS AND METHODS}

The present investigation was carried out at the experimental farm of Sakha Agricultural Research Station, Kafr El-Sheikh, Egypt, during the two successive seasons 2010/2011 and 2011/2012. Seven barley lines; $L_{1}, L_{2}, L_{3}$, $L_{4}, L_{5}, L_{6}$ and $L_{7}$ and three testers $T_{1}$ (Giza 129), $T_{2}$ (Giza 130) and $T_{3}$ (Giza 131) of hulless barley were used, the names and pedigrees of these genotypes are presented in Table (1).

Table (1): Names and pedigrees of parental barley genotypes.

\begin{tabular}{|c|c|c|}
\hline No & Genotypes & Pedigree \\
\hline \multicolumn{3}{|c|}{ Lines } \\
\hline 1 & L1 (Line-1) & CONGONA/3/ATACO/BERMEJO//HIGO/4/PETUNIA 1 \\
\hline 2 & $L_{2}$ (Line-2) & $\begin{array}{l}\text { LIGNEE640/P1382798//DC-B/3/MOLA/4/LION/10/CLN- } \\
\text { B/7/S.P-B/LIGNEE640/6/S.P-B/5/GLORIA-BAR/4/ } \\
\text { SOTOL//2762/BC-B/3/11012.2/TERN-B//H272/8/ FALCON- } \\
\text { BAR/9/ LION/11/PETUNIA 1 }\end{array}$ \\
\hline 3 & $L_{3}$ (Line-3) & CLN-B/80.5138//GLORIA-BAR/COPAL/3/CERRAJA \\
\hline 4 & -4 (Line-4) & $\begin{array}{l}\text { CONGONA/3/ATACO/ACHIRA//HIGO/7/ZARZA/5/ } \\
\text { GLORIA-BAR/4/SOTOL//2762/BC-B/3/11012.2/TERN- } \\
\text { B//H272/6/SEN }\end{array}$ \\
\hline 5 & L5 (Line-5) & PETUNIA 2 \\
\hline 6 & L6 (Line-6) & ALANDA//LIGNEE527//ARAR/3/BF891M-653 \\
\hline 7 & 17 (Line-7) & BF891M-597 \\
\hline \multicolumn{3}{|c|}{ Testers } \\
\hline 1 & $T_{1}$ (Giza 129) & Local Variety \\
\hline 2 & $T_{2}$ (Giza 130) & Local Variety \\
\hline 3 & $T_{3}$ (Giza 131) & Local Variety \\
\hline
\end{tabular}

In 2010/2011 season, the three testers were crossed with the seven lines to produce $F_{1}$ hybrids of 21 top crosses. In 2011/2012 season, $21 F_{1}$ hybrids, three testers and seven lines were planted in two experiments. The first 
experiment was given planting irrigation only (water stress condition, S). The second was irrigated three times after planting irrigation (normal condition, $\mathrm{N})$. Each experiment was designated in a randomized complete block design with three replicates. Each parent and $F_{1}$ was represented by two rows per replicate. Each row was $1.5 \mathrm{~m}$ long, and spaces between rows were $30 \mathrm{~cm}$ with $15 \mathrm{~cm}$ between plants. All the recommended agronomic practices for barley production were applied at the proper time. Ten guarded plants were randomly taken from each entry to collect data on plant height $(\mathrm{cm})$, spike length $(\mathrm{cm})$, number of spikes/plant (spike), number of grains /spike (grain), 100-grain weight $(\mathrm{g})$ and grain yield /plant $(\mathrm{g})$.

Statistical analysis:

A regular analysis of variance of a randomized complete block design was conducted. If the differences among genotypes, crosses and their parents being significant, Line $x$ tester analysis was performed according to Kempthorne (1957) and Singh and Chaudhary (1977). Heritability in broad $\left(\mathrm{H}^{2}\right)$ and narrow $\left(\mathrm{h}^{2}\right)$ senses were calculated according to Allard (1960) and Mather (1949). The phenotypic and genotypic coefficients of variation were estimated using the formulae developed by Burton (1952). Expected $(\Delta \mathrm{g})$ and predicted $\Delta \mathrm{g}(\%)$ genetic advance calculated as suggested by Borthakur and Poehllman (1970). Data of yield and some related traits were used to estimate the drought susceptibility index (DSI) as suggested by Fisther and Maurer (1978) as follows: DSI $=\left(1-Y_{d} / Y_{p}\right) / D$. Where: $Y_{d}=$ Performance of a genotype under drought stress, $Y_{p}=$ Performance of a genotype under normal irrigation, $D=$ drought stress intensity $=1$ - (mean $Y_{d}$ of all genotypes / mean $Y_{p}$ of all genotypes).

\section{RESULTS AND DISCUSSION}

Analysis of variance:

The estimation of the analysis of variance as shown in Table (2), revealed highly significant differences among genotypes for all studied traits. These results indicated that genotypic differences among genotypes were presented.

Table (2): Observed mean squares from ordinary analysis of variance for the studied traits under normal, stress and their combined analysis during $2011 / 2012$ season.

\begin{tabular}{|c|c|c|c|c|c|c|c|c|}
\hline \multirow{2}{*}{ S.O.V } & \multirow{2}{*}{\multicolumn{2}{|c|}{\begin{tabular}{|c|} 
df \\
SinqleComb
\end{tabular}}} & \multicolumn{3}{|c|}{ Plant height (cm) } & \multicolumn{3}{|c|}{ Spike length (cm) } \\
\hline & & & Normal & Stress & Comb. & Normal & Stress & Comb. \\
\hline Rep. & 2 & 5 & 35.45 & 13.3 & $291.93^{\star \star}$ & 0.34 & 1.4 & $2.71^{\star \star}$ \\
\hline Genotypes & 30 & 30 & $118.35^{\star *}$ & 123.8 & $166.16^{\star \star}$ & $2.63^{\star \star}$ & $8.06^{* *}$ & $4.47^{\star \star}$ \\
\hline $\begin{array}{l}\text { Parents } \\
\text { (P) }\end{array}$ & 9 & 9 & $79.12^{\star *}$ & $137.02^{\star *}$ & $148.31^{* *}$ & $2.98^{\star *}$ & $5.05^{*}$ & $4.62^{\star \star}$ \\
\hline $\begin{array}{c}\text { Crosses } \\
\text { (C) }\end{array}$ & 20 & 20 & $127.53^{\star *}$ & 119 & $164.33^{\star *}$ & $1.08^{\star *}$ & $8.37^{\star \star}$ & $1.75^{\star \star}$ \\
\hline P vs. C & 1 & 1 & $287.73^{\star \star}$ & $100.07^{\star}$ & $363.58^{* *}$ & $30.60^{\star \star}$ & $29.16^{\star *}$ & $57.53^{\star *}$ \\
\hline Lines (L) & 6 & 6 & $159.63^{\star \star}$ & $209.29^{\star \star}$ & $282.57^{\star \star}$ & $0.68^{*}$ & $11.78^{\star \star *}$ & $1.94^{* *}$ \\
\hline Testers (T) & 2 & 2 & $33.86^{\star \star}$ & $157.76^{\star \star}$ & $166.85^{\star \star}$ & $3.80^{* \star}$ & $12.59^{*}$ & $5.09^{\star \star}$ \\
\hline $\mathrm{LxT}$ & 12 & 12 & 127.10 & $67.52^{\star \star}$ & $104.79^{\star \star}$ & $0.83^{\star \star}$ & $5.96^{*}$ & $1.10^{*}$ \\
\hline Error & 60 & 150 & 28.53 & 22.02 & 35.42 & 0.28 & 0.8 & 0.56 \\
\hline
\end{tabular}

$\left(^{*}\right)$ and $\left(^{* *}\right)$ significant at 0.05 and 0.01 levels probability, respectively. 
Sultan, M. S. et al.

Table (2) continue:

\begin{tabular}{|c|c|c|c|c|c|c|c|c|}
\hline \multirow{3}{*}{$\begin{array}{c}\text { S.O.V } \\
\text { Rep. }\end{array}$} & \multicolumn{2}{|c|}{ df } & \multicolumn{3}{|c|}{ No. of spikes/plant (spike) } & \multicolumn{3}{|c|}{ No. of grains/spike (grain) } \\
\hline & Single & Comb. & Normal & Stress & Comb. & Normal & Stress & Comb. \\
\hline & 2 & 5 & $9.73^{* *}$ & 2.81 & $53.99^{* \star}$ & $8.13 \mathrm{~ns}$ & 58.11 & $62.11^{*}$ \\
\hline Genotypes & 30 & 30 & $14.06^{\star *}$ & $21.25^{\star \star}$ & $24.78^{\star \star}$ & $95.48^{\star \star}$ & $105.74^{\star *}$ & $171.14^{\star \star}$ \\
\hline Parents (P) & 9 & 9 & $13.10^{\star \star}$ & $7.92^{\star \star}$ & $16.81^{\star \star}$ & $50.80^{\star \star}$ & $67.87^{\star \star}$ & $81.60^{\star \star}$ \\
\hline $\begin{array}{c}\text { Crosses } \\
\text { (C) }\end{array}$ & 20 & 20 & $13.50^{\star *}$ & $21.85^{\star \star}$ & $22.22^{* *}$ & 9 ** & $47.38^{*}$ & $70.23^{\star *}$ \\
\hline P vs. C & 1 & 1 & $33.95^{\star \star}$ & $129.14^{\star *}$ & $147.75^{\star *}$ & $1385.6^{\star \star}$ & $1613.77^{* \star}$ & $2995.03^{\star \star}$ \\
\hline Lines (L) & 6 & 6 & $17.84^{\star \star}$ & $15.80^{\star \star}$ & $21.72^{\star \star}$ & $71.62^{\star \star}$ & $68.89^{\star \star}$ & $122.48^{\star \star}$ \\
\hline Testers (T) & 2 & 2 & $15.68^{* *}$ & $46.49^{\star \star}$ & $50.19^{\star \star}$ & $186.86^{\star \star}$ & $120.06^{\star \star}$ & $300.03^{\star *}$ \\
\hline LXT & 12 & 12 & $10.97^{* \star}$ & $20.77^{\star \star}$ & $17.81^{\star \star}$ & 18.19 & 24.51 & 5.81 \\
\hline Error & 60 & 150 & 2.36 & 2.82 & 4.18 & 13.73 & 14.28 & 22.49 \\
\hline
\end{tabular}

$\left(^{\star}\right)$ and $\left({ }^{* \star}\right)$ significant at 0.05 and 0.01 levels probability, respectively.

Table (2) continue:

\begin{tabular}{|c|c|c|c|c|c|c|c|c|}
\hline \multirow{2}{*}{ S.O.V } & \multirow{2}{*}{\multicolumn{2}{|c|}{\begin{tabular}{|c|} 
df \\
SingleComb \\
\end{tabular}}} & \multicolumn{3}{|c|}{ 100-grain weight (g) } & \multicolumn{3}{|c|}{ Grain yield/plant (g) } \\
\hline & & & Normal & Stress & Comb. & Normal & Stress & Comb. \\
\hline Rep. & 2 & 5 & 0.03 & 0.02 & $1.51^{\star \star}$ & $15.37^{\star}$ & 4.18 & $652.73^{\star \star}$ \\
\hline enotypes & 30 & 30 & $0.45^{\star *}$ & $0.56^{\star \star}$ & $0.81^{\star \star}$ & $150.70^{\star \star}$ & $166.20^{\star \star}$ & $248.62^{\star \star}$ \\
\hline $\begin{array}{r}\text { Pare } \\
(\mathbf{P}\end{array}$ & 9 & 9 & $0.41^{* *}$ & $0.31^{\star *}$ & $0.62^{* \star}$ & $61.87^{* *}$ & 33.20 & $73.97^{*}$ \\
\hline $\begin{array}{l}\text { Crosses } \\
\text { (C) }\end{array}$ & 20 & 20 & $0.37^{* *}$ & $0.57^{\star \star}$ & $0.69^{* \star}$ & $93.15^{\star \star}$ & $143.92^{\star *}$ & $42^{\star \star}$ \\
\hline P vs. C & 1 & 1 & $2.29^{\star \star}$ & $2.68^{\star \star}$ & $4.96^{* *}$ & $2101.31^{* *}$ & $9^{\star \star}$ & $3904.62^{* \star}$ \\
\hline & 6 & $\epsilon$ & 66 & 0.9 & $1.28^{\star \star}$ & 120. & $202.58^{\text {** }}$ & 192. \\
\hline ster & 2 & 2 & 54 & 1.3 & $1.74^{\star \star}$ & $124.90^{\star \star}$ & 50.11 & $155.03^{\star \star}$ \\
\hline$L x$ & 12 & 12 & $0.20^{\star *}$ & $0.27^{\star \star}$ & $0.22^{\star \star}$ & $74.26^{\star \star}$ & $130.23^{\star \star}$ & $118.52^{\star \star}$ \\
\hline iro & 60 & 50 & 0.03 & 0.09 & 0.09 & 4.82 & 4.59 & 29.42 \\
\hline
\end{tabular}

$\left({ }^{\star}\right)$ and $\left(^{\star \star}\right)$ significant at 0.05 and 0.01 levels probability, respectively.

Partitioning sum of squares due to genotypes (Table 2) revealed highly significant differences among parents, crosses and parents vs. crosses for all the studied traits, except grain yield/plant for parents under water stress. However, the crosses was further partitioned into lines, testers and line $x$ testers interaction. The mean squares of lines, testers and line $x$ testers showed significant or highly significant values for all the studied traits, except for plant height under normal condition, no. of grains/spike under the two conditions and their combined for line $x$ testers and grain yield/plant under stress for testers, These results indicated that lines differed in their order of performance in crosses with each of the testers.

\section{Mean performance of genotypes:}

Data in Table (3) indicated that the parental line-1 gave the highest values for spike length and no. of grains/spike under the two conditions and their combined, line-2 gave the highest values for no. of spikes/plant under stress condition, line- 4 gave the highest values for plant height and 100-grain weight under the two conditions and their combined, while line- 6 gave the highest values for grain yield/plant under the two conditions and their combined and no. of spikes/plant under normal condition and combined. 
Table (3): Mean performance of the barley genotypes for all studied traits under normal, stress and their combined data during 2011/2012 season.

\begin{tabular}{|c|c|c|c|c|c|c|c|}
\hline \multirow{2}{*}{\multicolumn{2}{|c|}{ Genotypes }} & \multicolumn{3}{|c|}{ Plant height (cm) } & \multicolumn{3}{|c|}{ Spike length (cm) } \\
\hline & & Normal & Stress & Comb. & Normal & Stress & Comb. \\
\hline \multicolumn{8}{|c|}{ Lines } \\
\hline \begin{tabular}{|l|}
1 \\
\end{tabular} & Line-1 & 112.0 & 110.3 & 111.2 & 9.67 & 9.00 & 9.34 \\
\hline 2 & Line-2 & 120.0 & 112.3 & 116.2 & 8.67 & 8.33 & 8.50 \\
\hline 3 & Line-3 & 118.0 & 107.7 & 112.8 & 8.67 & 8.00 & 8.34 \\
\hline 4 & Line-4 & 122.7 & 115.0 & 118.8 & 7.33 & 7.00 & 7.17 \\
\hline 5 & Line-5 & 110.0 & 104.7 & 107.3 & 8.33 & 7.67 & 8.00 \\
\hline 6 & Line-6 & 120.7 & 106.7 & 113.7 & 8.67 & 8.00 & 8.34 \\
\hline 7 & Line-7 & 118.3 & 116.7 & 117.5 & 9.33 & 8.33 & 8.83 \\
\hline \multicolumn{8}{|c|}{ Testers } \\
\hline 1 & Tester-1 & 109.3 & 102.3 & 105.8 & 9.67 & 9.33 & 9.50 \\
\hline 2 & Tester-2 & 120.0 & 113.7 & 116.8 & 10.67 & 9.33 & 10.00 \\
\hline 3 & Tester-3 & 123.3 & 117.0 & 120.2 & 10.33 & 10.00 & 10.17 \\
\hline \multicolumn{8}{|c|}{ Top crosses } \\
\hline 1 & $\mathrm{~L}_{1} \times \mathrm{T}_{1}$ & 126.7 & 119.0 & 122.8 & 10.00 & 9.67 & 9.84 \\
\hline 2 & $\mathrm{~L}_{1} \times \mathrm{T}_{2}$ & 115.7 & 110.3 & 113.0 & 11.00 & 10.33 & 10.67 \\
\hline 3 & $L_{1} \times T_{3}$ & 110.7 & 105.0 & 107.8 & 10.67 & 10.33 & 10.50 \\
\hline 4 & $\mathrm{~L}_{2} \times \mathrm{T}_{1}$ & 133.0 & 128.7 & 130.8 & 9.67 & 9.33 & 9.50 \\
\hline 5 & $\mathrm{~L}_{2} \times \mathrm{T}_{2}$ & 120.7 & 114.0 & 117.3 & 10.33 & 10.00 & 10.17 \\
\hline 6 & $\mathrm{~L}_{2} \times \mathrm{T}_{3}$ & 130.3 & 116.3 & 123.3 & 11.00 & 10.33 & 10.67 \\
\hline 7 & $\mathrm{~L}_{3} \times \mathrm{T}_{1}$ & 119.8 & 115.7 & 117.7 & 10.00 & 9.67 & 9.84 \\
\hline 8 & $\mathrm{~L}_{3} \times \mathrm{T}_{2}$ & 121.7 & 116.7 & 119.2 & 9.67 & 9.33 & 9.50 \\
\hline 9 & $\mathrm{~L}_{3} \times \mathrm{T}_{3}$ & 117.7 & 109.7 & 113.7 & 11.00 & 9.33 & 10.17 \\
\hline 10 & $\mathrm{~L}_{4} \times \mathrm{T}_{1}$ & 115.7 & 110.3 & 113.0 & 9.00 & 8.67 & 8.84 \\
\hline 11 & $\mathrm{~L}_{4} \times \mathrm{T}_{2}$ & 125.3 & 106.7 & 116.0 & 10.33 & 9.67 & 10.00 \\
\hline 12 & $\mathrm{~L}_{4} \times \mathrm{T}_{3}$ & 130.7 & 110.0 & 120.3 & 11.00 & 10.67 & 10.84 \\
\hline 13 & $\mathrm{~L}_{5} \times \mathrm{T}_{1}$ & 116.3 & 107.7 & 112.0 & 10.33 & 9.00 & 9.67 \\
\hline 14 & $\mathrm{~L}_{5} \times \mathrm{T}_{2}$ & 125.3 & 112.0 & 118.7 & 10.00 & 9.00 & 9.50 \\
\hline 15 & $\mathrm{~L}_{5} \times \mathrm{T}_{3}$ & 108.7 & 105.7 & 107.2 & 9.67 & 9.33 & 9.50 \\
\hline 16 & $\mathrm{~L}_{6} \times \mathrm{T}_{1}$ & 124.3 & 115.0 & 119.7 & 10.00 & 9.00 & 9.50 \\
\hline 17 & $\mathrm{~L}_{6} \times \mathrm{T}_{2}$ & 127.7 & 111.7 & 119.7 & 11.33 & 11.00 & 11.17 \\
\hline 18 & $\mathrm{~L}_{6} \times \mathrm{T}_{3}$ & 120.7 & 116.0 & 118.3 & 11.00 & 10.67 & 10.84 \\
\hline 19 & $L_{7} \times T_{1}$ & 120.3 & 108.0 & 114.2 & 10.33 & 10.00 & 10.17 \\
\hline 20 & $L_{7} \times T_{2}$ & 114.0 & 110.0 & 112.0 & 10.33 & 10.00 & 10.17 \\
\hline 21 & $\mathrm{~L}_{7} \times \mathrm{T}_{3}$ & 120.0 & 114.3 & 117.2 & 10.90 & 9.67 & 10.29 \\
\hline & D $\quad 0.05$ & 8.72 & 7.66 & 6.73 & 0.87 & 2.89 & 0.85 \\
\hline & 0.01 & 11.60 & 10.19 & 8.85 & 1.15 & 3.84 & 1.11 \\
\hline
\end{tabular}


Sultan, M. S. et al.

Table (3) Continue:

\begin{tabular}{|c|c|c|c|c|c|c|c|}
\hline \multirow{2}{*}{\multicolumn{2}{|c|}{ Genotypes }} & \multicolumn{3}{|c|}{ No. of spikes/plant (spike) } & \multicolumn{3}{|c|}{ No. of grains/spike (grain) } \\
\hline & & Normal & Stress & Comb. & Normal & Stress & omb. \\
\hline \multicolumn{8}{|c|}{ Lines } \\
\hline 1 & Line-1 & 15.44 & 12.11 & 13.78 & 70.00 & 68.00 & 69.00 \\
\hline 2 & Line-2 & 17.17 & 16.75 & 16.96 & 64.00 & 62.00 & 63.00 \\
\hline 3 & Line-3 & 19.83 & 15.08 & 17.46 & 68.00 & 62.00 & 65.00 \\
\hline 4 & Line-4 & 19.06 & 15.42 & 17.24 & 58.00 & 56.00 & 57.00 \\
\hline 5 & Line-5 & 18.48 & 16.06 & 17.27 & 64.00 & 56.00 & 60.00 \\
\hline 6 & Line-6 & 20.99 & 15.39 & 18.19 & 64.00 & 62.00 & 63.00 \\
\hline 7 & Line-7 & 18.82 & 15.28 & 17.05 & 68.00 & 58.00 & 63.00 \\
\hline \multicolumn{8}{|c|}{ Testers } \\
\hline 1 & Tester-1 & 14.08 & 12.64 & 13.36 & 70.00 & 66.00 & 68.00 \\
\hline 2 & Tester-2 & 17.11 & 12.42 & 14.77 & 68.00 & 66.00 & 67.00 \\
\hline 3 & Tester-3 & 16.51 & 15.08 & 15.80 & 72.00 & 70.00 & 71.00 \\
\hline \multicolumn{8}{|c|}{ Top crosses } \\
\hline 1 & $\mathrm{~L}_{1} \times \mathrm{T}_{1}$ & 17.00 & 15.42 & 16.21 & 72.00 & 70.00 & 71.00 \\
\hline 2 & $\mathrm{~L}_{1} \times \mathrm{T}_{2}$ & 14.67 & 12.67 & 13.67 & 78.00 & 74.00 & 76.00 \\
\hline 3 & $\mathrm{~L}_{1} \times \mathrm{T}_{3}$ & 18.22 & 18.00 & 18.11 & 80.00 & 72.00 & 76.00 \\
\hline 4 & $\mathrm{~L}_{2} \times \mathrm{T}_{1}$ & 17.44 & 17.17 & 17.31 & 74.00 & 70.00 & 72.00 \\
\hline 5 & $\mathrm{~L}_{2} \times \mathrm{T}_{2}$ & 18.83 & 16.83 & 17.83 & 78.00 & 76.00 & 77.00 \\
\hline 6 & $\mathrm{~L}_{2} \times \mathrm{T}_{3}$ & 18.08 & 15.17 & 16.63 & 82.00 & 74.00 & 78.00 \\
\hline 7 & $\mathrm{~L}_{3} \times \mathrm{T}_{1}$ & 23.30 & 18.22 & 20.76 & 72.00 & 66.00 & 69.00 \\
\hline 8 & $\mathrm{~L}_{3} \times \mathrm{T}_{2}$ & 20.00 & 14.33 & 17.17 & 72.00 & 70.00 & 71.00 \\
\hline 9 & $\mathrm{~L}_{3} \times \mathrm{T}_{3}$ & 20.00 & 15.67 & 17.84 & 74.00 & 68.00 & 71.00 \\
\hline 10 & $\mathrm{~L}_{4} \times \mathrm{T}_{1}$ & 17.00 & 16.22 & 16.61 & 68.00 & 66.00 & 67.00 \\
\hline 11 & $\mathrm{~L}_{4} \times \mathrm{T}_{2}$ & 20.00 & 12.00 & 16.00 & 80.00 & 70.00 & 75.00 \\
\hline 12 & $\mathrm{~L}_{4} \times \mathrm{T}_{3}$ & 20.00 & 19.33 & 19.67 & 76.00 & 74.00 & 75.00 \\
\hline 13 & $\mathrm{~L}_{5} \times \mathrm{T}_{1}$ & 19.11 & 17.67 & 18.39 & 68.00 & 66.00 & 67.00 \\
\hline 14 & $\mathrm{~L}_{5} \times \mathrm{T}_{2}$ & 16.56 & 14.17 & 15.37 & 74.00 & 70.00 & 72.00 \\
\hline 15 & $\mathrm{~L}_{5} \times \mathrm{T}_{3}$ & 21.33 & 16.42 & 18.88 & 68.00 & 66.00 & 67.00 \\
\hline 16 & $\mathrm{~L}_{6} \times \mathrm{T}_{1}$ & 18.67 & 18.33 & 18.50 & 74.00 & 73.33 & 73.67 \\
\hline 17 & $\mathrm{~L}_{6} \times \mathrm{T}_{2}$ & 20.67 & 20.11 & 20.39 & 80.00 & 78.00 & 79.00 \\
\hline 18 & $\mathrm{~L}_{6} \times \mathrm{T}_{3}$ & 20.33 & 16.33 & 18.33 & 78.00 & 76.00 & 77.00 \\
\hline 19 & $\mathrm{~L}_{7} \times \mathrm{T}_{1}$ & 22.00 & 21.83 & 21.92 & 72.00 & 70.00 & 71.00 \\
\hline 20 & $\mathrm{~L}_{7} \times \mathrm{T}_{2}$ & 16.00 & 15.66 & 15.83 & 76.00 & 72.00 & 74.00 \\
\hline 21 & $\mathrm{~L}_{7} \times \mathrm{T}_{3}$ & 20.67 & 17.11 & 18.89 & 76.00 & 74.00 & 75.00 \\
\hline & $\begin{array}{ll} & 0.05 \\
\end{array}$ & 2.51 & 2.74 & 2.31 & 6.05 & 6.17 & 5.37 \\
\hline & 0.01 & 3.34 & 3.64 & 3.04 & 8.05 & 8.21 & 7.05 \\
\hline
\end{tabular}


Table (3) Continue:

\begin{tabular}{|c|c|c|c|c|c|c|c|}
\hline \multirow{2}{*}{\multicolumn{2}{|c|}{ Genotypes }} & \multicolumn{3}{|c|}{ 100-grain weight $(\mathrm{g})$} & \multicolumn{3}{|c|}{ Grain yield/plant (g) } \\
\hline & & Normal & Stress & Comb. & Normal & Stress & Comb. \\
\hline \multicolumn{8}{|c|}{ Lines } \\
\hline \begin{tabular}{|l|l}
1 & \\
\end{tabular} & Line-1 & 4.40 & 4.10 & 4.25 & 30.94 & 24.40 & 27.67 \\
\hline \begin{tabular}{|l|l}
2 \\
\end{tabular} & Line-2 & 3.86 & 3.57 & 3.72 & 27.55 & 24.15 & 25.85 \\
\hline \begin{tabular}{|l|l}
3 \\
\end{tabular} & Line-3 & 4.29 & 3.65 & 3.97 & 37.46 & 23.11 & 30.29 \\
\hline \begin{tabular}{|l|l}
4 \\
\end{tabular} & Line-4 & 4.68 & 4.35 & 4.52 & 33.55 & 27.95 & 30.75 \\
\hline 5 & Line-5 & 4.40 & 3.81 & 4.11 & 33.75 & 22.16 & 27.96 \\
\hline \begin{tabular}{|l|l}
6 \\
\end{tabular} & Line-6 & 4.42 & 4.07 & 4.25 & 38.57 & 29.45 & 34.01 \\
\hline \begin{tabular}{|l|l}
7 & \\
\end{tabular} & Line-7 & 3.84 & 3.79 & 3.82 & 31.90 & 28.82 & 30.36 \\
\hline \multicolumn{8}{|c|}{ Testers } \\
\hline \begin{tabular}{|l|l}
1 & \\
\end{tabular} & Tester-1 & 3.82 & 3.30 & 3.56 & 24.43 & 19.71 & 22.07 \\
\hline 2 & Tester-2 & 4.49 & 4.26 & 4.38 & 33.88 & 23.30 & 28.59 \\
\hline 3 & Tester-3 & 4.91 & 3.98 & 4.45 & 37.95 & 30.62 & 34.29 \\
\hline \multicolumn{8}{|c|}{ Top crosses } \\
\hline \begin{tabular}{|l|l|}
1 \\
\end{tabular} & $\mathrm{~L}_{1} \times \mathrm{T}_{1}$ & 4.91 & 4.58 & 4.75 & 39.08 & 32.22 & 35.65 \\
\hline 2 & $\mathrm{~L}_{1} \times \mathrm{T}_{2}$ & 4.78 & 4.68 & 4.73 & 35.59 & 30.34 & 32.97 \\
\hline 3 & $\mathrm{~L}_{1} \times \mathrm{T}_{3}$ & 4.66 & 4.30 & 4.48 & 44.00 & 40.23 & 42.12 \\
\hline 4 & $\mathrm{~L}_{2} \times \mathrm{T}_{1}$ & 4.20 & 3.88 & 4.04 & 35.13 & 31.03 & 33.08 \\
\hline \begin{tabular}{|l|}
5 \\
\end{tabular} & $\mathrm{~L}_{2} \times \mathrm{T}_{2}$ & 4.57 & 4.39 & 4.48 & 43.58 & 36.78 & 40.18 \\
\hline 6 & $\mathrm{~L}_{2} \times \mathrm{T}_{3}$ & 4.40 & 3.95 & 4.18 & 42.19 & 28.81 & 35.50 \\
\hline 7 & $\mathrm{~L}_{3} \times \mathrm{T}_{1}$ & 4.50 & 4.13 & 4.32 & 49.04 & 32.68 & 40.86 \\
\hline 8 & $\mathrm{~L}_{3} \times \mathrm{T}_{2}$ & 5.13 & 4.03 & 4.58 & 47.94 & 27.84 & 37.89 \\
\hline 9 & $\mathrm{~L}_{3} \times \mathrm{T}_{3}$ & 4.62 & 3.95 & 4.29 & 44.29 & 27.27 & 35.78 \\
\hline 10 & $\mathrm{~L}_{4} \times \mathrm{T}_{1}$ & 4.56 & 3.94 & 4.25 & 34.24 & 33.15 & 33.70 \\
\hline 11 & $\mathrm{~L}_{4} \times \mathrm{T}_{2}$ & 4.79 & 4.74 & 4.77 & 49.65 & 25.88 & 37.77 \\
\hline 12 & $\mathrm{~L}_{4} \times \mathrm{T}_{3}$ & 5.05 & 4.81 & 4.93 & 49.80 & 48.26 & 49.03 \\
\hline 13 & $\mathrm{~L}_{5} \times \mathrm{T}_{1}$ & 5.02 & 3.60 & 4.31 & 48.30 & 27.14 & 37.72 \\
\hline 14 & $\mathrm{~L}_{5} \times \mathrm{T}_{2}$ & 4.84 & 4.21 & 4.53 & 38.53 & 27.25 & 32.89 \\
\hline 15 & $\mathrm{~L}_{5} \times \mathrm{T}_{3}$ & 4.78 & 4.50 & 4.64 & 44.93 & 35.20 & 40.07 \\
\hline 16 & $\mathrm{~L}_{6} \times \mathrm{T}_{1}$ & 4.60 & 4.23 & 4.42 & 41.28 & 36.96 & 39.12 \\
\hline 17 & $\mathrm{~L}_{6} \times \mathrm{T}_{2}$ & 4.81 & 4.75 & 4.78 & 51.57 & 50.47 & 51.02 \\
\hline 18 & $\mathrm{~L}_{6} \times \mathrm{T}_{3}$ & 4.93 & 4.76 & 4.85 & 50.84 & 38.42 & 44.63 \\
\hline 19 & $\mathrm{~L}_{7} \times \mathrm{T}_{1}$ & 3.60 & 3.41 & 3.51 & 37.12 & 34.94 & 36.03 \\
\hline 20 & $\mathrm{~L}_{7} \times \mathrm{T}_{2}$ & 4.72 & 3.80 & 4.26 & 37.29 & 36.24 & 36.77 \\
\hline 21 & $\mathrm{~L}_{7} \times \mathrm{T}_{3}$ & 4.13 & 4.11 & 4.12 & 42.11 & 36.49 & 39.30 \\
\hline LS & 0.05 & 0.30 & 0.50 & 0.34 & 3.58 & 9.60 & 6.14 \\
\hline LS & 0.01 & 0.40 & 0.66 & 0.44 & 4.77 & 12.77 & 8.07 \\
\hline
\end{tabular}

\section{Combining ability analysis:}

For testers; $T_{2}$ (Giza 130) gave the highest values for spike length, no. of spikes/plant under normal condition and 100-grain weight under stress condition, while $T_{3}$ (Giza 131) gave the highest values for plant height, no. of grains/spike, grain yield/plant under the two conditions and their combined, spike length, no. of spikes/plant under stress condition and combined and 100-grain weight under normal condition and combined. For crosses; top crosses no. 4 for plant height under the two conditions and their combined, 6 for no. of grains/spike under normal, cross no. 7 for no. of spikes/plant under normal condition, cross no. 8 for 100-grain weight under normal condition, 
cross no. 12 for 100-grain weight under stress condition and combined and grain yield/plant under normal condition, cross no. 17 for spike length under the two conditions and their combined, no. of grains/spike and grain yield/plant under stress condition and combined and cross no. 19 for no. of spikes/plant under stress condition and combined gave the highest values for these traits.

Data in Table (4) indicated that, general combining ability (GCA) expressed main effects and specific combining ability (SCA) expressed interactions. GCA/SCA ratio was used as a measure to understand the nature of gene action involved.

Table (4): Analysis of variance for general (GCA), specific (SCA) combining ability and GCA/SCA under normal, stress and their combined analysis during 2011/2012 season.

\begin{tabular}{|c|c|c|c|c|c|c|c|}
\hline traits & & $\begin{array}{c}\text { Plant } \\
\text { height } \\
\text { (cm) }\end{array}$ & $\begin{array}{c}\text { Spike } \\
\text { length } \\
\text { (cm) }\end{array}$ & $\begin{array}{l}\text { No. of } \\
\text { spikes/ } \\
\text { plant } \\
\text { (spike) }\end{array}$ & $\begin{array}{l}\text { No. of } \\
\text { grains/ } \\
\text { spike } \\
\text { (grain) }\end{array}$ & $\begin{array}{c}\text { 100- } \\
\text { grain } \\
\text { weight } \\
\text { (g) }\end{array}$ & $\begin{array}{c}\text { Grain } \\
\text { yield/ } / \\
\text { plant (g) }\end{array}$ \\
\hline \multirow{3}{*}{$\sigma^{2} \mathrm{GCA}$} & Normal & 0.22 & 0.01 & 0.09 & 0.95 & 0.00 & 0.65 \\
\hline & Stress & 1.61 & 0.07 & 0.05 & 0.49 & 0.01 & 0.62 \\
\hline & Combined & 0.96 & 0.01 & 0.07 & 0.92 & 0.01 & 0.46 \\
\hline \multirow{3}{*}{$\sigma^{2} \mathrm{SCA}$} & Normal & 32.86 & 18.00 & 2.87 & 1.49 & 0.05 & 23.15 \\
\hline & Stress & 15.17 & 0.94 & 5.98 & 6.33 & 0.06 & 31.88 \\
\hline & Combined & 11.56 & 0.09 & 2.27 & 2.78 & 0.02 & 14.85 \\
\hline \multirow{3}{*}{ Error term } & Normal & 9.51 & 0.09 & 0.79 & 4.58 & 0.01 & 1.61 \\
\hline & Stress & 7.34 & 0.27 & 0.94 & 4.76 & 0.03 & 11.53 \\
\hline & Combined & 5.90 & 0.09 & 0.70 & 3.75 & 0.01 & 4.90 \\
\hline \multirow{3}{*}{$\sigma^{2} \mathrm{GCA} / \sigma^{2} \mathrm{SCA}$} & Normal & -0.03 & 0.00 & -0.03 & 0.10 & -0.02 & 0.00 \\
\hline & Stress & -0.06 & -0.02 & -0.01 & -0.23 & -0.07 & -0.04 \\
\hline & Combined & -0.07 & 1.95 & -0.03 & 0.24 & -0.11 & -0.04 \\
\hline
\end{tabular}

The ratio of GCA/SCA were lesser than unity for all the studied traits under both conditions except for spike length under combined analysis which mean that non-additive gene effects played an important role in the inheritance of these traits. In such cases, a bulk method would be fruitful to eliminate the effect of dominance in the advanced generation. These results agreed with those obtained by Amer (2010), Eid (2010) and Amer (2011).

\section{Estimates of general combining ability effects (GCA):}

Data in Table (5) indicated that, the parental line-1 exhibited desirable significant positive GCA for plant height, spike length under stress condition and 100-grain weight under the two conditions and their combined. Meaning that, this genotype could be considered as good combiner for these traits. The parental line-2 gave significant or highly significant positive GCA for plant height under the two conditions and their combined and spike length under stress condition. The parental line-3 was a good combiner for no. of spikes/plant and grain yield/plant under normal condition. line-4 was a good combiner for 100-grain weight under the two conditions and their combined. Line-5 was a good combiner for 100 -grain weight under normal condition. 
line-6 was a good combiner for spike length, no. of grains/spike, 100-grain weight and grain yield/plant under the two conditions and their combined and no. of spikes/plant under stress condition and combined. line-7 was a good combiner for no. of spikes/plant under stress condition and combined. So, these lines could be used as parent for the development of desirable hybrids. These results are in agreement with those obtained by Budak (2000), Amer (2010), Eid (2010) and Amer (2011) where they observed significant and positive GCA for these traits in their respective studies.

Table (5): Estimates of general combining ability effects for the studied traits under normal, stress and their combined analysis during 2011/2012 season.

\begin{tabular}{|c|c|c|c|c|c|c|}
\hline \multirow{2}{*}{ Parents } & \multicolumn{3}{|c|}{ Plant height $(\mathrm{cm})$} & \multicolumn{3}{|c|}{ Spike length (cm) } \\
\hline & Normal & Stress & Comb. & Normal & Stress & Comb. \\
\hline & & & & & & \\
\hline $\begin{array}{ll}L_{1} \\
\end{array}$ & -3.53 & $4.38^{* *}$ & 0.43 & 0.20 & $0.76^{*}$ & 0.23 \\
\hline $\mathrm{L}_{2}$ & $6.80^{\star \star}$ & $7.38^{\star \star}$ & $7.09^{\star \star}$ & -0.03 & $0.76^{*}$ & 0.12 \\
\hline$L_{3}$ & -1.49 & 1.49 & 0.00 & -0.14 & 0.21 & -0.21 \\
\hline $\mathrm{L}_{4}$ & 2.69 & -1.84 & 0.43 & -0.25 & $-1.79^{* \star}$ & -0.05 \\
\hline $\mathrm{L}_{5}$ & $-4.42^{*}$ & $-6.84^{\star \star}$ & $-5.63^{\star \star}$ & $-0.36^{*}$ & $-1.35^{\star}$ & $-0.60^{* *}$ \\
\hline $\mathrm{L}_{6}$ & 3.03 & -1.06 & 0.98 & $0.42^{*}$ & $0.87^{* *}$ & $0.40^{*}$ \\
\hline $\mathrm{L}_{7}$ & -3.08 & $-3.51^{*}$ & $-3.30^{*}$ & 0.16 & 0.54 & 0.10 \\
\hline $\begin{array}{l}\text { LSD } \\
0.05\end{array}$ & 3.56 & 3.13 & 2.75 & 0.35 & 0.58 & 0.35 \\
\hline 0.01 & 4.74 & 4.16 & 3.61 & 0.47 & 0.78 & 0.46 \\
\hline Testers & & & & & & \\
\hline$T_{1}$ & 1.11 & $2.81^{\star *}$ & $1.96^{*}$ & $-0.46^{\star \star}$ & 0.16 & $-0.39^{\star \star}$ \\
\hline$T_{2}$ & 0.28 & -0.14 & 0.07 & 0.07 & $0.68^{* *}$ & 0.13 \\
\hline$T_{3}$ & -1.39 & $-2.67^{*}$ & $-2.03^{*}$ & $0.39^{\star \star}$ & $-0.84^{*}$ & $0.27^{\star}$ \\
\hline $\begin{array}{l}\text { LSD } \\
0.05\end{array}$ & 2.33 & 2.05 & 1.80 & 0.23 & 0.48 & 0.23 \\
\hline 0.01 & 3.1 & 2.72 & 2.37 & 0.31 & 0.63 & 0.30 \\
\hline
\end{tabular}

$\left(^{*}\right)$ and $\left(^{* *}\right)$ significant at 0.05 and 0.01 levels probability, respectively.

Table (5)continue:

\begin{tabular}{|c|c|c|c|c|c|c|}
\hline \multirow[b]{2}{*}{ Parents } & \multicolumn{3}{|c|}{ No. of spikes/plant (spike) } & \multicolumn{3}{|c|}{ No. of grains/spike (grain) } \\
\hline & Normal & Stress & Comb. & Normal & Stress & Comb. \\
\hline \multicolumn{7}{|l|}{ Lines } \\
\hline $\mathrm{L}_{1}$ & $-2.41^{\star \star}$ & $-1.12^{*}$ & $-1.76^{\star \star}$ & 1.81 & -0.44 & 0.68 \\
\hline $\mathrm{L}_{2}$ & -0.92 & -0.75 & -0.84 & $3.14^{*}$ & 0.89 & 2.02 \\
\hline $\mathrm{L}_{3}$ & $2.06^{* *}$ & -1.07 & 0.49 & -2.19 & $-3.78^{*}$ & $-2.98^{\star *}$ \\
\hline $\mathbf{L}_{4}$ & -0.04 & 0.71 & 0.33 & -0.19 & 0.22 & 0.02 \\
\hline $\mathrm{L}_{5}$ & -0.04 & -1.06 & -0.55 & $-4.86^{\star \star}$ & -3.11 & $-3.98^{* *}$ \\
\hline L6 & 0.85 & $1.12^{*}$ & $0.98^{*}$ & $2.48^{*}$ & $4.00^{*}$ & $3.24^{\star *}$ \\
\hline $\mathrm{L}_{7}$ & 0.51 & $2.17^{\star \star}$ & $1.34^{\star \star}$ & -0.19 & 2.22 & 1.02 \\
\hline LSD 0.05 & 1.02 & 1.12 & 0.94 & 2.47 & 3.49 & 2.19 \\
\hline 0.01 & 1.36 & 1.49 & 1.24 & 3.29 & 4.64 & 2.88 \\
\hline \multicolumn{7}{|l|}{ Testers } \\
\hline$T_{1}$ & 0.18 & $1.41^{\star \star}$ & $0.79^{\star}$ & $-3.43^{\star *}$ & $-2.63^{*}$ & $-3.03^{* *}$ \\
\hline$T_{2}$ & $-0.94^{\star \star}$ & $-1.56^{\star \star}$ & $-1.25^{\star \star}$ & $2.00^{*}$ & 2.03 & $2.02^{\star \star}$ \\
\hline$T_{3}$ & $0.76^{*}$ & 0.15 & 0.46 & 1.43 & 0.60 & 1.02 \\
\hline LSD 0.05 & 0.67 & 0.73 & 0.62 & 1.62 & 2.29 & 1.43 \\
\hline 0.01 & 0.89 & 0.97 & 0.81 & 2.15 & 3.04 & 1.88 \\
\hline
\end{tabular}

$\left(^{*}\right)$ and $\left(^{* *}\right)$ significant at 0.05 and 0.01 levels probability, respectively. 
Sultan, M. S. et al.

Table (5)continue:

\begin{tabular}{|c|c|c|c|c|c|c|}
\hline \multirow[b]{2}{*}{ Parents } & \multicolumn{3}{|c|}{ 100-grain weight (g) } & \multicolumn{3}{|c|}{ Grain yield/plant (g) } \\
\hline & Normal & Stress & Comb. & Normal & Stress & Comb. \\
\hline \multicolumn{7}{|l|}{ Lines } \\
\hline $\mathrm{L}_{1}$ & $0.14^{*}$ & $0.36^{* *}$ & $0.25^{\star \star}$ & $-3.61^{* *}$ & -0.34 & -1.97 \\
\hline $\mathrm{L}_{2}$ & $-0.26^{\star \star}$ & -0.19 & $-0.22^{* \star}$ & $-2.87^{\star \star}$ & -2.4 & $-2.63^{*}$ \\
\hline$L_{3}$ & 0.10 & $-0.22^{*}$ & -0.06 & $3.92^{\star \star}$ & $-5.34^{* \star}$ & -0.71 \\
\hline $\mathrm{L}_{4}$ & $0.15^{\star}$ & $0.24^{*}$ & $0.20^{* \star}$ & 1.39 & 3.51 & 2.45 \\
\hline $\mathrm{L}_{5}$ & $0.23^{\star *}$ & -0.15 & 0.04 & 0.75 & $-4.74^{*}$ & -1.99 \\
\hline $\mathrm{L}_{6}$ & $0.13^{*}$ & $0.37^{* *}$ & $0.25^{\star *}$ & $4.73^{\star \star}$ & $8.02^{* \star}$ & $6.37^{* *}$ \\
\hline $\mathrm{L}_{7}$ & $-0.49^{* *}$ & $-0.42^{* *}$ & $-0.46^{* \star}$ & $-4.32^{\star *}$ & 1.29 & -1.52 \\
\hline LSD 0.05 & 0.12 & 0.20 & 0.14 & 1.46 & 3.92 & 2.51 \\
\hline 0.01 & 0.16 & 0.27 & 0.18 & 1.95 & 5.21 & 3.29 \\
\hline \multicolumn{7}{|l|}{ Testers } \\
\hline$T_{1}$ & $-0.16^{\star \star}$ & $-0.29^{* *}$ & $-0.23^{\star \star}$ & $-2.57^{\star \star}$ & -1.01 & $-1.79^{*}$ \\
\hline $\mathbf{T}_{2}$ & $0.16^{\star *}$ & $0.18^{\star \star}$ & $0.17^{\star \star}$ & 0.28 & -0.77 & -0.24 \\
\hline$T_{3}$ & 0.01 & 0.11 & 0.06 & $2.29^{\star *}$ & 1.78 & $2.03^{*}$ \\
\hline LSD 0.05 & 0.08 & 0.13 & 0.09 & 0.96 & 2.57 & 1.64 \\
\hline 0.01 & 0.11 & 0.18 & 0.12 & 1.27 & 3.41 & 2.16 \\
\hline
\end{tabular}

$\left({ }^{\star}\right)$ and $\left({ }^{*}\right)$ significant at 0.05 and 0.01 levels probability, respectively.

For testers; as shown in Table (5) the parental tester-1 showed desirable significant positive GCA for plant height and no. of spikes/plant under stress condition and combined. The parental tester- 2 was a good combiner for 100-grain weight under the two conditions and their combined, spike length under stress condition and no. of grains/spike under normal condition and combined. The parental tester-3 was a good combiner for spike length, grain yield/plant under normal condition and combined and no. of spikes/plant under normal condition.

\section{Estimates of specific combining ability effects (SCA):}

Data in Table (6) indicated that, for plant height, the top crosses no. 4 under stress condition, no. 1 under normal condition and combined and no. 14 under normal condition and combined expressed significant and positive SCA. However, the combinations showed significant positive SCA may be useful in exploitation of heterosis due to their desirable plant height. For spike length the top crosses no. 13 under normal condition, no. 3, 6, 10, 11 and 18 under stress condition showed significant positive value for SCA. Regarding to no. of spikes/plant, significant positive SCA were showed for crosses no. 7, 11 under normal condition, no. 19 under normal condition and combined, no. $3,5,17$ under stress condition and combined, no. 10 and 12 under stress condition. For 100-grain weight, the top crosses no. 1 under normal and combined, no. 8, 12, 13, 20 under normal condition, no. 7 and 21 under stress expressed significant positive SCA. With respect to grain yield/plant, positive and significant SCA effects were revealed by the top crosses no. 12, 17 under the two conditions and their combined, 7 under normal condition and combined, no. 5, 11 and 13 under normal condition. These results indicated that non-additive gene effects were predominant in these particular combinations of barley hybrids for grain yield/plant due to the presence of considerable inter and intra-allelic interactions. Therefore, these top crosses might be interest in breeding program and could be utilized for increasing grain yield/plant following approach of gene accumulation for the characters. These results are in general agreement with those reported by Sharma et al (2003), Mahmoud (2006), El-Sayed (2007), Katta et al (2009), Amer (2010), Eid (2010) and Amer (2011). 
Table (6): Estimates of specific combining ability effects for the studied traits under normal, stress and their combined analysis during 2011/2012 season.

\begin{tabular}{|c|c|c|c|c|c|c|c|c|}
\hline \multirow{2}{*}{\multicolumn{3}{|c|}{ Top crosses }} & \multicolumn{3}{|c|}{ Plant height (cm) } & \multicolumn{3}{|c|}{ Spike length $(\mathrm{cm})$} \\
\hline & & & Normal & Stress & Comb. & Normal & Stress & Comb. \\
\hline \multirow{3}{*}{ Line-1 } & Giza 129 & 1 & $7.89^{*}$ & -0.14 & 3.88 & -0.10 & -0.38 & 0.01 \\
\hline & Giza 130 & 2 & -2.28 & 4.14 & 0.93 & 0.38 & -0.57 & 0.15 \\
\hline & Giza 131 & 3 & -5.61 & -4.00 & $-4.81^{*}$ & -0.28 & $0.95^{\star \star}$ & -0.15 \\
\hline \multirow{3}{*}{ Line-2 } & Giza 129 & 4 & 3.89 & $7.19^{*}$ & $5.54^{*}$ & -0.21 & -0.38 & -0.05 \\
\hline & Giza 130 & 5 & $-7.61^{\star}$ & -3.52 & $-5.57^{\star}$ & -0.07 & -0.57 & -0.07 \\
\hline & Giza 131 & 6 & 3.72 & -3.67 & 0.03 & 0.28 & $0.95^{\star \star}$ & 0.12 \\
\hline \multirow{3}{*}{ Line-3 } & Giza 129 & 7 & -1.03 & 1.08 & 0.03 & 0.23 & -0.16 & 0.28 \\
\hline & Giza 130 & 8 & 1.68 & 0.03 & 0.86 & $-0.62^{*}$ & -0.35 & -0.24 \\
\hline & Giza 131 & 9 & -0.65 & -1.11 & -0.88 & 0.39 & 0.51 & -0.04 \\
\hline \multirow{3}{*}{ Line-4 } & Giza 129 & 10 & $-9.33^{* \star}$ & 4.08 & -2.62 & $-0.66^{*}$ & $1.84^{\star \star}$ & -0.38 \\
\hline & Giza 130 & 11 & 1.16 & -3.3 & -1.07 & 0.15 & $1.32^{* \star}$ & -0.24 \\
\hline & Giza 131 & 12 & $8.16^{*}$ & -0.70 & 3.69 & 0.5 & $-3.16^{\star *}$ & $0.62^{*}$ \\
\hline \multirow{3}{*}{ Line-5 } & Giza 129 & 13 & -1.55 & -3.59 & -2.57 & $0.79^{*}$ & 0.73 & 0.51 \\
\hline & Giza 130 & 14 & $8.28^{\star \star}$ & 3.70 & $5.99^{*}$ & -0.07 & 0.21 & -0.18 \\
\hline & Giza 131 & 15 & $-6.72^{\star}$ & -0.11 & -3.42 & $-0.72^{*}$ & $-0.94^{\star \star}$ & -0.32 \\
\hline \multirow{3}{*}{ Line-6 } & Giza 129 & 16 & -1.00 & -2.03 & -1.51 & -0.32 & $-1.49^{\star \star}$ & $-0.66^{\star}$ \\
\hline & Giza 130 & 17 & 3.16 & -2.41 & 0.38 & 0.49 & 0.32 & $0.65^{*}$ \\
\hline & Giza 131 & 18 & -2.17 & 4.44 & 1.14 & -0.17 & $1.17^{* \star}$ & 0.01 \\
\hline \multirow{3}{*}{ Line-7 } & Giza 129 & 19 & 1.12 & $-6.59^{*}$ & -2.74 & 0.27 & -0.16 & 0.30 \\
\hline & Giza 130 & 20 & -4.39 & 1.37 & -1.51 & -0.26 & -0.35 & -0.06 \\
\hline & Giza 131 & 21 & 3.28 & 5.22 & 4.25 & -0.01 & 0.51 & -0.24 \\
\hline \multicolumn{3}{|c|}{ LSD 0.05} & 6.17 & 5.42 & 4.76 & 0.61 & 0.79 & 0.60 \\
\hline \multicolumn{3}{|c|}{0.01} & 8.2 & 7.21 & 6.26 & 0.82 & 0.93 & 0.79 \\
\hline
\end{tabular}

$\left(^{\star}\right)$ and $\left({ }^{\star *}\right)$ significant at 0.05 and 0.01 levels probability, respectively.

Table (6) Continue.:

\begin{tabular}{|c|c|c|c|c|c|c|c|c|}
\hline \multirow{2}{*}{\multicolumn{3}{|c|}{ Top crosses }} & \multicolumn{3}{|c|}{ No. of spikes/plant (spike) } & \multicolumn{3}{|c|}{ No. of grains/spike (grain) } \\
\hline & & & Normal & Stress & Comb. & Normal & Stress & Comb. \\
\hline \multirow{3}{*}{ Line-1 } & Giza 129 & 1 & 0.19 & $-2.02^{*}$ & -0.91 & -1.24 & 1.97 & 0.37 \\
\hline & Giza 130 & 2 & -1.02 & -1.81 & -1.42 & -0.67 & -0.70 & -0.68 \\
\hline & Giza 131 & 3 & 0.83 & $3.82^{\star \star}$ & $2.33^{* \star}$ & 1.90 & -1.27 & 0.32 \\
\hline \multirow{3}{*}{ Line-2 } & Giza 129 & 4 & -0.85 & -0.63 & -0.74 & -0.57 & -1.37 & -0.97 \\
\hline & Giza 130 & 5 & 1.65 & $2.00^{*}$ & $1.83^{*}$ & -2.00 & 1.97 & -0.02 \\
\hline & Giza 131 & 6 & -0.8 & -1.37 & -1.09 & 2.57 & -0.60 & 0.98 \\
\hline \multirow{3}{*}{ Line-3 } & Giza 129 & 7 & $2.02^{*}$ & 0.74 & 1.38 & 2.76 & -0.70 & 1.03 \\
\hline & Giza 130 & 8 & -0.16 & -0.19 & -0.17 & -2.67 & 2.63 & -0.02 \\
\hline & Giza 131 & 9 & $-1.86^{*}$ & -0.56 & -1.21 & -0.1 & -1.94 & -1.02 \\
\hline \multirow{3}{*}{ Line-4 } & Giza 129 & 10 & $-2.18^{*}$ & $1.96^{*}$ & -0.11 & -3.24 & 3.30 & 0.03 \\
\hline & Giza 130 & 11 & $1.94^{*}$ & $-4.30^{\star *}$ & -1.18 & 3.33 & -5.37 & -1.02 \\
\hline & Giza 131 & 12 & 0.24 & $2.33^{*}$ & 1.29 & -0.10 & 2.06 & 0.98 \\
\hline \multirow{3}{*}{ Line-5 } & Giza 129 & 13 & -0.07 & 0.17 & 0.05 & 1.43 & -1.37 & 0.03 \\
\hline & Giza 130 & 14 & -1.50 & -0.36 & -0.93 & 2.00 & -2.03 & -0.02 \\
\hline & Giza 131 & 15 & 1.57 & 0.19 & 0.88 & -3.43 & 3.40 & -0.02 \\
\hline \multirow{3}{*}{ Line-6 } & Giza 129 & 16 & -1.4 & -1.33 & -1.37 & 0.10 & -1.14 & -0.52 \\
\hline & Giza 130 & 17 & 1.72 & $3.41^{* *}$ & $2.56^{\star \star}$ & 0.67 & 2.86 & 1.76 \\
\hline & Giza 131 & 18 & -0.32 & $-2.07^{\star}$ & -1.20 & -0.76 & -1.71 & -1.24 \\
\hline \multirow{3}{*}{ Line-7 } & Giza 129 & 19 & $2.27^{*}$ & 1.11 & $1.69^{*}$ & 0.76 & -0.70 & 0.03 \\
\hline & Giza 130 & 20 & $-2.62^{\star *}$ & 1.24 & -0.69 & -0.67 & 0.63 & -0.02 \\
\hline & Giza 131 & 21 & 0.35 & $-2.35^{\star}$ & -1.00 & -0.10 & 0.06 & -0.02 \\
\hline \multicolumn{3}{|c|}{ LSD 0.05} & 1.77 & 1.94 & 1.64 & 4.28 & 6.05 & 3.79 \\
\hline \multicolumn{3}{|c|}{0.01} & 2.36 & 2.58 & 2.15 & 5.69 & 8.04 & 4.99 \\
\hline
\end{tabular}

$\left({ }^{\star}\right)$ and $\left(^{* \star}\right)$ significant at 0.05 and 0.01 levels probability, respectively. 
Sultan, M. S. et al.

Table (6) Continue:

\begin{tabular}{|c|c|c|c|c|c|c|c|c|}
\hline \multirow{2}{*}{\multicolumn{3}{|c|}{ Top crosses }} & \multicolumn{3}{|c|}{100 -grain weight $(\mathrm{g})$} & \multicolumn{3}{|c|}{ Grain yield/plant $(g)$} \\
\hline & & & Normal & Stress & Comb. & Normal & Stress & Comb. \\
\hline \multirow{3}{*}{ Line-1 } & Giza 129 & 1 & $0.29^{* *}$ & 0.25 & $0.27^{*}$ & 2.09 & -1.04 & 0.53 \\
\hline & Giza 130 & 2 & -0.16 & 0.19 & 0.01 & $-4.25^{\star \star}$ & -3.15 & -3.70 \\
\hline & Giza 131 & 3 & -0.13 & $-0.43^{*}$ & $-0.28^{*}$ & 2.16 & 4.19 & 3.17 \\
\hline \multirow{3}{*}{ Line-2 } & Giza 129 & 4 & -0.03 & 0.1 & 0.04 & $-2.60^{*}$ & -0.17 & -1.39 \\
\hline & Giza 130 & 5 & 0.02 & 0.14 & 0.08 & $2.99^{\star}$ & 5.35 & 4.17 \\
\hline & Giza 131 & 6 & 0.00 & -0.24 & -0.12 & -0.4 & -5.18 & -2.79 \\
\hline \multirow{3}{*}{ Line-3 } & Giza 129 & 7 & -0.09 & $0.39^{*}$ & 0.15 & $4.52^{\star \star}$ & 4.43 & $4.47^{*}$ \\
\hline & Giza 130 & 8 & $0.22^{*}$ & -0.19 & 0.02 & 0.57 & -0.65 & -0.04 \\
\hline & Giza 131 & 9 & -0.13 & -0.2 & -0.17 & $-5.09^{\star \star}$ & -3.77 & $-4.43^{*}$ \\
\hline \multirow{3}{*}{ Line-4 } & Giza 129 & 10 & -0.07 & -0.27 & -0.17 & $-7.76^{\star \star}$ & 3.09 & -2.33 \\
\hline & Giza 130 & 11 & -0.17 & 0.06 & -0.05 & $4.80^{\star \star}$ & $-11.46^{\star *}$ & -3.33 \\
\hline & Giza 131 & 12 & $0.24^{*}$ & 0.21 & 0.22 & $2.95^{\star}$ & $8.37^{*}$ & $5.66^{*}$ \\
\hline \multirow{3}{*}{ Line-5 } & Giza 129 & 13 & $0.30^{* \star}$ & -0.22 & 0.04 & $6.95^{\star \star}$ & -1.71 & 2.62 \\
\hline & Giza 130 & 14 & -0.19 & -0.07 & -0.13 & $-5.68^{\star \star}$ & -1.84 & -3.76 \\
\hline & Giza 131 & 15 & -0.10 & 0.29 & 0.09 & -1.27 & 3.55 & 1.14 \\
\hline \multirow{3}{*}{ Line-6 } & Giza 129 & 16 & -0.02 & -0.11 & -0.06 & $-4.05^{\star \star}$ & -4.65 & $-4.35^{\star}$ \\
\hline & Giza 130 & 17 & -0.13 & 0.09 & -0.02 & $3.39^{* *}$ & $10.63^{* *}$ & $7.01^{* *}$ \\
\hline & Giza 131 & 18 & 0.14 & 0.02 & 0.08 & 0.66 & -5.97 & -2.66 \\
\hline \multirow{3}{*}{ Line-7 } & Giza 129 & 19 & $-0.39^{\star \star}$ & -0.14 & $-0.26^{*}$ & 0.85 & 0.06 & 0.45 \\
\hline & Giza 130 & 20 & $0.41^{\star *}$ & -0.22 & 0.10 & -1.83 & 1.13 & -0.35 \\
\hline & Giza 131 & 21 & -0.03 & $0.36^{*}$ & 0.17 & 0.98 & -1.18 & -0.10 \\
\hline \multicolumn{3}{|c|}{ LSD 0.05} & 0.21 & 0.35 & 0.24 & 2.53 & 6.79 & 4.34 \\
\hline \multicolumn{3}{|c|}{0.01} & 0.28 & 0.47 & 0.31 & 3.37 & 9.03 & 5.70 \\
\hline
\end{tabular}

$\left({ }^{*}\right)$ and $\left(^{* *}\right)$ significant at 0.05 and 0.01 levels probability, respectively.

Coefficients of variability:

As shown in Table (7), it could be concluded that the (PCV and GCV) were low in magnitude for plant height, no. of grains/spike and 100-grain weight. While, it was observed that the phenotypic (PCV) and genotypic (GCV) coefficients of variability were high in magnitudes for no. of spikes/plant and grain yield/plant under both conditions and their combined data. These results are in general agreement with those reported by ElSayed (2012). 
Table (7): Estimates of the genetic variance components for all the studied traits under normal, stress and their combined analysis during 2011/2012 season.

\begin{tabular}{|c|c|c|c|c|c|c|c|c|}
\hline Characters & aramete & $\mathrm{s}^{2} \mathrm{ph}$ & $\mathbf{s}^{2} \mathbf{G}$ & $s^{2} E$ & $\mathbf{s}^{2} A$ & $\mathbf{s}^{2} D$ & PCV \% & GCV \% \\
\hline \multirow{3}{*}{$\begin{array}{l}\text { Plant height } \\
(\mathrm{cm})\end{array}$} & Normal & 42.8 & 33.29 & 9.51 & 0.43 & 32.86 & 5.45 & 4.81 \\
\hline & Stress & 25.73 & 18.39 & 7.34 & 3.22 & 15.17 & 4.53 & 3.83 \\
\hline & Comb. & 25.28 & 13.48 & 11.81 & 1.92 & 11.56 & 4.34 & 3.17 \\
\hline \multirow{3}{*}{$\begin{array}{c}\text { Spike length } \\
\text { (cm) }\end{array}$} & Normal & 0.28 & 0.19 & 0.09 & 0.01 & 0.18 & 5.34 & 4.36 \\
\hline & Stress & 1.35 & 1.08 & 0.27 & 0.14 & 0.94 & 12.51 & 11.1 \\
\hline & Comb. & 0.29 & 0.11 & 0.18 & 0.02 & 0.09 & 5.61 & 3.37 \\
\hline \multirow{3}{*}{$\begin{array}{l}\text { No. of spikes } \\
\text { plant (spike) }\end{array}$} & Normal & 3.83 & 3.04 & 0.79 & 0.17 & 2.87 & 10.5 & 9.36 \\
\hline & Stress & 7.01 & 6.07 & 0.94 & 0.09 & 5.98 & 16.59 & 15.44 \\
\hline & Comb. & 3.80 & 2.41 & 1.39 & 0.14 & 2.27 & 11.28 & 8.98 \\
\hline \multirow{3}{*}{$\begin{array}{l}\text { No. of grains } \\
\text { spike (grain) }\end{array}$} & Normal & 7.96 & 3.38 & 4.58 & 1.89 & 1.49 & 3.91 & 2.55 \\
\hline & Stress & 12.07 & 7.31 & 4.76 & 0.98 & 6.33 & 5.08 & 3.95 \\
\hline & Comb. & 12.11 & 4.61 & 7.50 & 1.83 & 2.78 & 4.95 & 3.05 \\
\hline \multirow{3}{*}{$\begin{array}{l}\text { 100-grains } \\
\text { weight }(g)\end{array}$} & Normal & 0.07 & 0.06 & 0.01 & 0.01 & 0.05 & 5.76 & 5.26 \\
\hline & Stress & 0.1 & 0.07 & 0.03 & 0.01 & 0.06 & 7.6 & 6.28 \\
\hline & Comb. & 0.06 & 0.0 & 0.03 & 0.01 & 0.02 & 5.74 & 4.13 \\
\hline \multirow{3}{*}{$\begin{array}{l}\text { Grain yield/ } \\
\text { plant }(\mathbf{g})\end{array}$} & Normal & 26.04 & 24.44 & 1.60 & 1.29 & 23.15 & 12.79 & 12.39 \\
\hline & Stress & 44.64 & 33.11 & 11.53 & 1.23 & 31.88 & 21.33 & 18.37 \\
\hline & Comb. & 25.58 & 15.77 & 9.81 & 0.92 & 14.85 & 14.20 & 11.15 \\
\hline
\end{tabular}

\section{Heritability estimates:}

The heritability estimates either in narrow sense is important to plant breeders since they judge the expected improvement that could be obtained through selection programs. Broad sense heritability values were found to be moderate to high in magnitudes for all the studied traits and ranged from $36.14 \%$ for spike length under combined to $93.84 \%$ for grain yield/plant under normal condition (Table, 8).

Table (8): Estimates heritability and genetic advance for all studied traits under normal, stress and their combined analysis during 2011/2012 season.

\begin{tabular}{|c|c|c|c|c|c|}
\hline Characters & Parameter & $\mathbf{H}^{2} \%$ & $h^{2} \%$ & $\Delta \mathbf{g}$ & $\Delta \mathbf{g} \%$ \\
\hline \multirow{3}{*}{ Plant height } & Normal & 77.78 & 1.01 & 0.14 & 0.11 \\
\hline & Stress & 71.47 & 12.52 & 1.31 & 1.17 \\
\hline & Comb. & 53.30 & 7.57 & 0.78 & 0.68 \\
\hline \multirow{3}{*}{ Spike length } & Normal & 66.78 & 2.47 & 0.03 & 0.27 \\
\hline & Stress & 80.15 & 9.9 & 0.24 & 2.53 \\
\hline & Comb. & 36.14 & 5.45 & 0.06 & 0.63 \\
\hline \multirow{3}{*}{$\begin{array}{l}\text { No. of spikes/ } \\
\text { plant }\end{array}$} & Normal & 79.47 & 4.47 & 0.18 & 0.97 \\
\hline & Stress & 86.61 & 1.28 & 0.07 & 0.44 \\
\hline & Comb. & 63.40 & 3.66 & 0.15 & 0.85 \\
\hline \multirow{3}{*}{$\begin{array}{l}\text { No. of grains/ } \\
\text { spike }\end{array}$} & Normal & 42.47 & 23.78 & 1.38 & 1.91 \\
\hline & Stress & 60.56 & 8.1 & 0.58 & 0.85 \\
\hline & Comb. & 38.09 & 15.14 & 1.09 & 1.54 \\
\hline \multirow{3}{*}{$\begin{array}{c}\text { 100-grains } \\
\text { weight }\end{array}$} & Normal & 83.41 & 4.39 & 0.02 & 0.52 \\
\hline & Stress & 68.37 & 10.2 & 0.07 & 1.6 \\
\hline & Comb. & 51.89 & 16.22 & 0.08 & 1.92 \\
\hline \multirow{3}{*}{$\begin{array}{l}\text { Grain yield/ } \\
\text { plant }\end{array}$} & Normal & 93.84 & 4.95 & 0.52 & 1.3 \\
\hline & Stress & 74.17 & 2.76 & 0.38 & 1.21 \\
\hline & Comb. & 61.66 & 3.60 & 0.38 & 1.05 \\
\hline
\end{tabular}


Sultan, M. S. et al.

Narrow sense heritability was generally low to moderate and ranged from $1.01 \%$ for plant height under normal condition to $23.78 \%$ for no. of grains/spike under normal condition. The high difference between broad and narrow sense heritability estimates which was found for all the studied traits was an expected due to more important of non-additive genes in the inheritance of these traits as it mentioned before. These results are in line with those reported by El-Sayed (2007), El-Shawy (2008) and El-Sayed (2012).

\section{Genetic advance under selection:}

Genetic advance was calculated using phenotypic standard deviation and heritability in narrow sense (Table 8). Relatively high genetic advance was found in association with high heritability in narrow sense values for plant height, spike length under stress condition, no. of grains/spike under normal condition and combined, 100-grain weight under stress condition and combined and grain yield/plant under the two conditions and their combined analysis. Therefore, selection for these studied traits could be more effective than the other traits because of its high expected $(\Delta \mathrm{g} \%)$ genetic advance which reflected in high genetic variability. On the other hand, relative low genetic advance was associated with low heritability values for plant height, spike length under normal and combined, no. of spikes/plant under the two conditions and their combined, no. of grains/spike under stress condition and 100-grain weight under normal indicating that selection for these studied traits would be less successful than for the former cases. These results in agreement with those obtained by El-Sayed (2007), El-Shawy (2008) and ElSayed (2012).

\section{Drought susceptibility index (DSI):}

A drought susceptibility index (SI), which provides a measure of stress resistance based on minimization of yield loss under stress as compared to optimum conditions, rather than on yield level under stress, has been used to characterize the relative drought tolerance of wheat genotypes (Fisher and Maurer, 1978). This index was used to estimate the relative stress loss because it accounted for variation in yield potential and stress intensity. This index could be estimated based on many traits. Lower stress susceptibility index than unity $(\mathrm{Sl}<1)$ is synonymous to high stress tolerance, while high stress susceptibility index $(S I>1)$ mean higher stress sensitivity.

Data in Table (9) indicated that, for parents; line-2, line-4, tester-1 and tester3 were the best where it possessed DSI less than one for yield and most of its components, revealing that these parents were more resistance to water stress. For hybrids, the crosses no. 1, 2, 3, 4, 5, 10, 12, 16, 17, 19, 20 and 21 were more resistant to water stress condition where it possessed DSI less than one for yield and most of its components, revealing that these crosses were more resistant to water stress. 
Table (9): Drought susceptibility index for barley parents and their $F_{1}$ crosses based on all studied traits during 2011/2012 season.

\begin{tabular}{|c|c|c|c|c|c|c|c|}
\hline \multicolumn{2}{|l|}{ Genotypes } & $\begin{array}{c}\text { Plant } \\
\text { height } \\
\text { (cm) }\end{array}$ & $\begin{array}{c}\text { Spike } \\
\text { length } \\
(\mathrm{cm})\end{array}$ & $\begin{array}{c}\text { No. of } \\
\text { spikes/ } \\
\text { plant } \\
\text { (spike) }\end{array}$ & $\begin{array}{l}\text { No. of } \\
\text { grains/ } \\
\text { spike } \\
\text { (grain) }\end{array}$ & $\begin{array}{l}100- \\
\text { grain } \\
\text { weight } \\
\text { (g) }\end{array}$ & $\begin{array}{c}\text { Grain } \\
\text { yield/ } \\
\text { plant (g) }\end{array}$ \\
\hline \multicolumn{8}{|l|}{ Lines } \\
\hline Line-1 & 1 & 0.22 & 1.13 & 1.51 & 0.55 & 0.73 & 0.99 \\
\hline Line-2 & 2 & 0.95 & 0.64 & 0.17 & 0.60 & 0.81 & 0.58 \\
\hline Line-3 & 3 & 1.30 & 1.26 & 1.68 & 1.69 & 1.60 & 1.79 \\
\hline Line-4 & 4 & 0.93 & 0.74 & 1.34 & 0.66 & 0.76 & 0.78 \\
\hline Line-5 & 5 & 0.72 & 1.29 & 0.92 & 2.40 & 1.44 & 1.60 \\
\hline Line-6 & 6 & 1.72 & 1.26 & 1.87 & 0.60 & 0.85 & 1.10 \\
\hline Line-7 & 7 & 0.21 & 1.75 & 1.32 & 2.82 & 0.14 & 0.45 \\
\hline \multicolumn{8}{|l|}{ Testers } \\
\hline Tester-1 & 1 & 0.95 & 0.57 & 0.72 & 1.10 & 1.46 & 0.90 \\
\hline Tester-2 & 2 & 0.78 & 2.05 & 1.92 & 0.56 & 0.55 & 1.46 \\
\hline Tester-3 & 3 & 0.76 & 0.52 & 0.61 & 0.53 & 2.04 & 0.90 \\
\hline \multicolumn{8}{|l|}{ Top crosses } \\
\hline L1 $x$ T1 & 1 & 0.90 & 0.54 & 0.65 & 0.53 & 0.72 & 0.82 \\
\hline L1 $\times$ T2 & 2 & 0.69 & 0.99 & 0.95 & 0.98 & 0.23 & 0.69 \\
\hline L1 $\times$ T3 & 3 & 0.76 & 0.52 & 0.08 & 1.92 & 0.83 & 0.40 \\
\hline L2 $\times$ T1 & 4 & 0.48 & 0.57 & 0.11 & 1.04 & 0.82 & 0.54 \\
\hline L2 $\times$ T2 & 5 & 0.82 & 0.52 & 0.74 & 0.49 & 0.42 & 0.73 \\
\hline L2 $\times$ T3 & 6 & 1.60 & 0.99 & 1.13 & 1.87 & 1.10 & 1.48 \\
\hline L3 $\times$ T1 & 7 & 0.51 & 0.54 & 1.53 & 1.60 & 0.88 & 1.56 \\
\hline L3 $\times$ T2 & 8 & 0.61 & 0.57 & 1.98 & 0.53 & 2.31 & 1.95 \\
\hline L3 $\times$ T3 & 9 & 1.01 & 2.48 & 1.52 & 1.56 & 1.56 & 1.79 \\
\hline L4 x T1 & 10 & 0.69 & 0.60 & 0.32 & 0.56 & 1.46 & 0.15 \\
\hline L4 $\times$ T2 & 11 & 2.21 & 1.04 & 2.80 & 2.40 & 0.11 & 2.23 \\
\hline L4 $\times$ T3 & 12 & 2.35 & 0.49 & 0.23 & 0.50 & 0.51 & 0.14 \\
\hline L5 $\times$ T1 & 13 & 1.11 & 2.10 & 0.53 & 0.56 & 3.04 & 2.04 \\
\hline L5 $\times$ T2 & 14 & 1.58 & 1.63 & 1.01 & 1.04 & 1.40 & 1.36 \\
\hline L5 $\times$ T3 & 15 & 0.41 & 0.57 & 1.61 & 0.56 & 0.63 & 1.01 \\
\hline L6 $\times$ T1 & 16 & 1.11 & 1.63 & 0.13 & 0.17 & 0.87 & 0.49 \\
\hline L6 $\times$ T2 & 17 & 1.86 & 0.48 & 0.19 & 0.48 & 0.13 & 0.10 \\
\hline L6 $\times$ T3 & 18 & 0.57 & 0.49 & 1.38 & 0.49 & 0.37 & 1.14 \\
\hline L7 $\times$ T1 & 19 & 1.52 & 0.52 & 0.05 & 0.53 & 0.57 & 0.27 \\
\hline L7 $\times$ T2 & 20 & 0.52 & 0.52 & 0.15 & 1.01 & 2.10 & 0.13 \\
\hline L7 $\times$ T3 & 21 & 0.70 & 1.84 & 1.21 & 0.50 & 0.05 & 0.62 \\
\hline
\end{tabular}

\section{REFERENCES}

Allard, A. M. (1960). Principles of plant breeding. Jon Wily and Sons. Inc. NY, U. S. A., p. 92.

Amer, Kh. A. (2010). Inheritance of drought tolerance in some barley genotypes. Egypt. J. Agric. Res., (1) 88: 89-106.

Amer, Kh. A. (2011). Genetic Analysis of yield and its components under normal and drought conditions in some barley crosses. Egypt. J. Plant Breed (15) 2: 65:79. 
Blum, A. (1979). Genetic improvement of drought resistance in crop plants. A case for sorghum. pp: 495-545. In: H. Hussell and R.C. Staples (ed.). Stress Physiology in Crop Plants. Wiley Interscience, New York.

Borthakur, D.N. and J.M. Poehlman (1970). Heritability and genetic advance for kernel weight in barley. Crop Sci. 10: 452-453.

Budak, N. (2000). Heterosis, general and specific combining ability estimates at $F_{1}$ and $F_{2}$ generations of a $8 \times 8$ diallel cross population of barley. Turkish J. of Field Crops. Society of Field Crop Sci., Izmir, Turkey. 5 (2): 61-70.

Burton, G.W. (1952). Quantitative inheritance in grasses. Proc. $6^{\text {th }}$ Int. Grassid Congr. 1: 270-283.

Eid, A. A. (2010). The genetic behavior for salinity tolerance in some barley genotypes. Egypt. J. Agric. Res., (1) 88: 67-86.

El-Sayed, M. M. A. (2007). Estimation of quantitative genetic statistical in diallel crosses of barley. M. Sc. Thesis Fac., Agric., Kafr El-Sheikh, Tanta Univ., Egypt.

El-Sayed, M. M. A. (2012). Genetical studies on some barley genotypes and its tolerance to drought. Ph. D. Thesis Fac., Agric., Tanta Univ., Egypt.

El-Shawy, E. E. A. (2008). Genetic analysis of some important traits of sixrowed barley in normal and saline affected fields. M. Sc. Thesis, Fac. Agric., Kafr El-Sheikh, Tanta Univ., Egypt.

Finlay, K.W. (1968). The significance of adaptation in wheat breeding. pp: 742-754. In: Proc. 3 ${ }^{\text {rd }}$ Int. Wheat Genetics Symp., 5-9 August, Australian Academy of Sciences, Canberra, A.C.T.

Finlay, K.W. and G.N. Wilkinson (1963). The analysis of adaptation in plant breeding programmes. Aust. J. Agric. Res., 14: 742-754.

Fisher, R.A. and R. Maurer (1978). Drought resistance in spring wheat cultivars I. Grain yield responses. Aust. J. Agric. Res., 29:897-912.

Katta, Y.S., A.A. Eid., M.S. Abd El-Aty and Sally, M. EL-Wakeel (2009). Studies on tolerance of some hulless barley crosses to drought. $6^{\text {th }}$ International Plant Bred. Conf., Ismalia, Egypt, 3-5 May 2009. 867:885.

Kempthorne, O. (1957). An introduction to genetic statistics. lowa State Univ., John Wiley and Sons Inc., New York, USA. 545 pp.

Mather, K. (1949). Biometrical Genetics. Dover Publications, Inc., London.

Mahmoud, Badeaa A. M. (2006). Genetic evaluation of some barley traits in crosses under saline and non-saline conditions. M. Sc. thesis, Fac. Agric., Kafr El-Sheikh, Tanta Univ., Egypt.

Sharma, Y., S. N. Sharma., P. Joshi and R. S. Sain (2003). Combining ability in the $F_{1}$ and $F_{2}$ generations of a diallel cross in six rowed barley (Hordeum vulgare L.). Acta Agron. Hungaricae., 51: 281-288.

Singh, R.K. and B.D. Chaudhary (1977). Biometrical methods in quantitative genetic analysis. Kalyani, Publishers, Daragnai, New Delhi.

Smith, E.L. (1982). Heat and drought tolerant wheats of the future. pp: 141147. In: Proc. of the National Wheat Res. Conf. USA-ARS, Beltville, Maryland.

Sojka, R.E.; L.H. Stolzy and R.A. Fischer (1981). Seasonal drought response of selected wheat cultivars. Agron. J., 73: 838-845. 


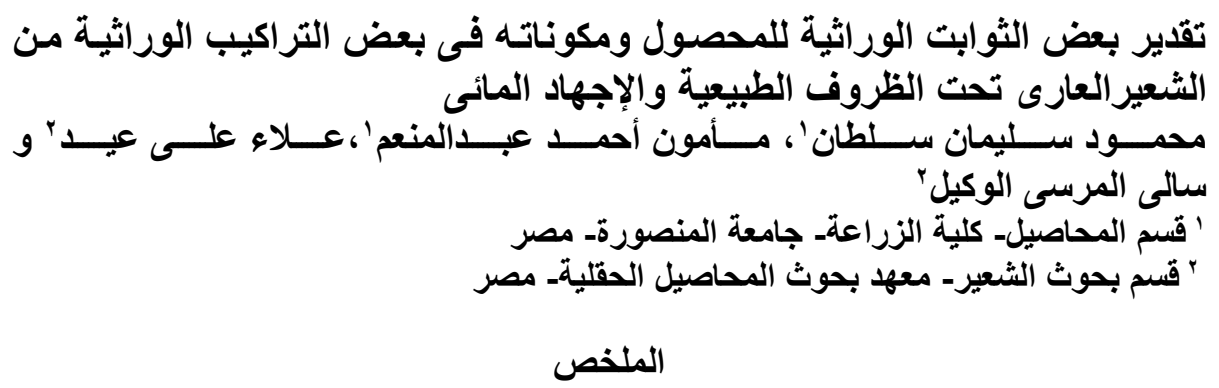

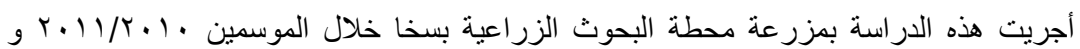

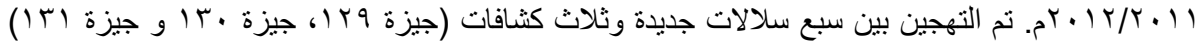

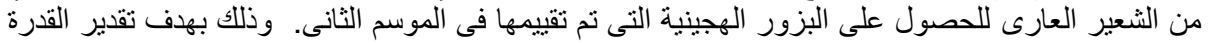

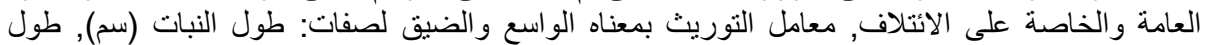

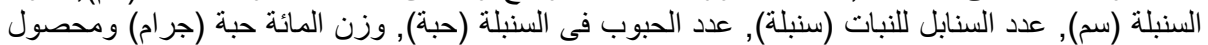

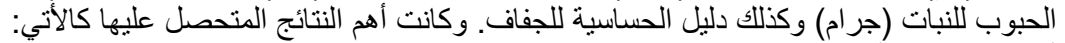

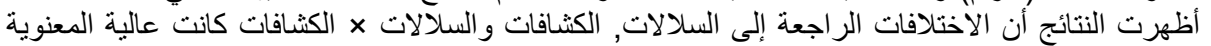

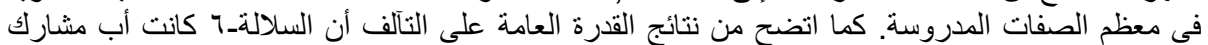

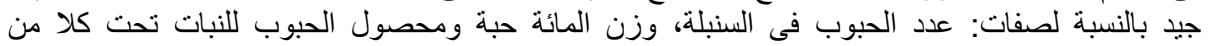

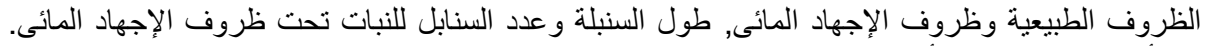

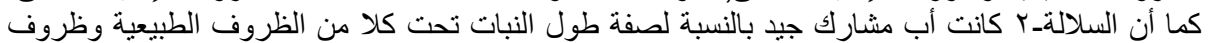

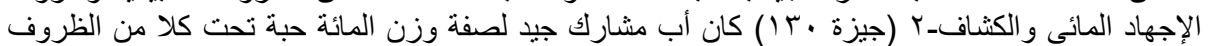

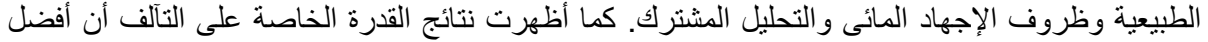

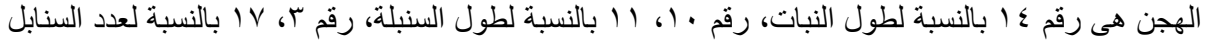

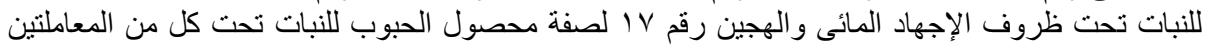

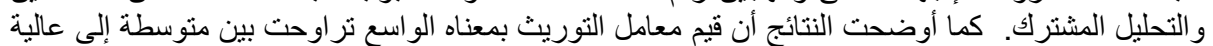

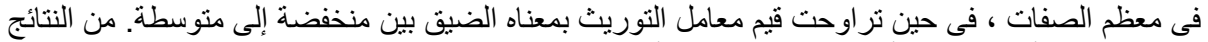

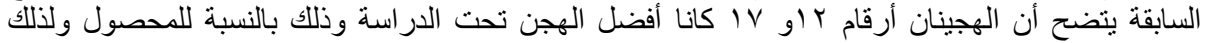

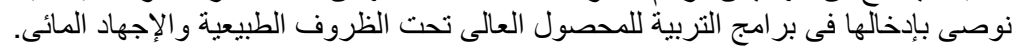

كلية الزراعة - جامعة المنصورة

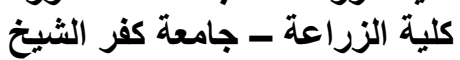

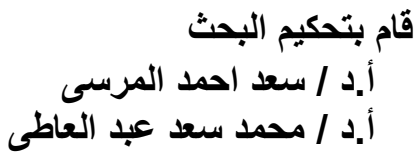

